\section{Reply to Ross' commentary: Reproductive benefit through newborn screening: preferences, policy and ethics}

European Journal of Human Genetics (2012) 20, 486-487; doi:10.1038/ejhg.2012.25; published online 29 February 2012

In her commentary to our article, ${ }^{1}$ Lainie Friedman Ross offers three main criticisms of our work. First, she suggests that the health care providers (HCPs) in our study may not have been arguing that the generation of reproductive risk information is a primary benefit of newborn screening (NBS). Second, she reviews evidence on the reproductive behaviors of parents who are provided with infant carrier information through NBS to suggest a disconnect between parental preferences and the goals of HCPs. Finally, she suggests that the distinctive features of sickle cell trait (SCT) complicate our discussion of reproductive benefits, limiting the clarity and generalizability of our findings. ${ }^{2}$ We welcome the opportunity to respond.

The main goal of our article was to suggest that providers do indeed perceive the incidental generation of reproductive risk information through NBS as one of its primary benefits. This assertion derives from their dominant rationale that identification of reproductive risk through NBS is an inherent - inextricably linked - consequence of NBS and, in turn, the disclosure of reproductive risk information facilitates disease prevention, one of the main goals of NBS. ${ }^{1}$ Dr Ross refers to this assertion as a 'free' benefit - hence, ancillary - but indeed HCPs conceived it as primary. As one of our HCP participants put it:

Identifying somebody who actually has a disease to me is probably even less important than actually trying to prevent the disease... preventing disease and providing information for the patients who do develop disease is a very important part of this. That may not be the original purpose or what the original purpose of newborn screening but again newborn screening was set up around PKU (phenylketonuria) and hypothyroidism so... ${ }^{1}$

That HCPs were expressing this viewpoint can also be inferred from our survey data, as there was less support for screening for the purpose of clinical benefit (55-56\% agree or strongly agree to NBS enabling presymptomatic diagnosis for treatment, or in the absence of treatment) compared with screening for the purpose of reproductive benefit (68-78\% agree or strongly agree to identifying the infant's carrier status and parent's reproductive risks). ${ }^{1}$ Providers' views that reproductive benefit serves a primary role in NBS appear to align with the arguments of some commentators, who have called for an expanded valuation of what have traditionally been viewed as the secondary benefits of NBS. ${ }^{3-7}$ However, as we suggested in our article, these views are misaligned with the principles that provide warrant for population screening, ${ }^{8-10}$ and with ethical and policy norms guiding the realization of reproductive benefit in other population screening contexts. ${ }^{7,11}$ As such, and here we agree wholeheartedly with Dr Ross, they are cause for some concern.

Dr Ross reviews largely epidemiological evidence on disease incidence associated with the receipt of infant carrier information through NBS to point to the limited use of this 'reproductive benefit', and to suggest a disconnect between parental preferences and the goals of HCPs that we identify. Yet behavior is not synonymous with preferences, as evidenced by a substantial literature demonstrating the disconnect between genetic testing intentions and actual uptake. ${ }^{12-14}$ The literature about parental attitudes regarding infant carrier information through NBS points to a similar disconnect as, notwithstanding epidemiological evidence regarding behavior, most parents state that they feel better knowing their child's carrier status, ${ }^{15,16}$ and want this information to know their own and their family members' reproductive risks. ${ }^{17}$ This literature thus suggests that parental 'preferences' are at least somewhat aligned with those of HCPs. (Of course, whether parents view this as a primary benefit is not explored in existing studies.)

Finally, Dr Ross suggests that the distinctive features of SCT complicate our discussion of reproductive benefits. Dr Ross questions whether HCPs' support for the identification of SCT stemmed from its 'not so benign' nature. The policy implications of the potential clinical significance of SCT have been previously debated publicly. ${ }^{18,19}$ Further, as our earlier article ${ }^{20}$ on the complex and sometimes dissonant interpretations of SCT pointed out, the guidance provided to families and providers by NBS programs in the United States and United Kingdom are contradictory. Indeed, what any health implications mean or should mean for NBS policy is unclear. Thus, the goal in our current article is not to rehash these complex issues but to focus on the one issue raised by SCT that is unquestioned - its relevance to reproductive risk. We are confident that, in our qualitative interviews, the issue of reproductive benefit was discussed independently of the potential health significance of sickle cell disorder (SCD) carrier status. Dr Ross ends with a call for fuller engagement with the communities who are disproportionately affected by SCD (and SCT), to assess whether this reproductive 'benefit' is of real or only hypothetical interest. Yet as we have noted above, practices and preferences are often unrelated. Indeed, our argument was not that these are (or should be) tangible and realizable benefits, but rather, that HCPs perceive them as such. This is, in our view, a notable finding: one that warrants careful consideration given its potential implications for screening practice and policy.

We would thus agree that our study identifies a need for engagement with HCPs about 'the ethically justifiable public health goals of a universal NBS program'. Yet, given the increasing alignment between HCPs' views, parental preferences and some policy statements in this domain, we suggest broader engagement with all relevant communities, including lay/citizens, parents, HCPs and decision makers, to ensure that the goals and directions of NBS programs strike an appropriate balance between benefits to screened individuals and those to families and societies, in the context of public health programs.

\section{CONFLICT OF INTEREST}

The authors declare no conflict of interest.

Yvonne Bombard ${ }^{1}$ and Fiona A Miller ${ }^{2}$ ${ }^{1}$ Department of Public Health and Epidemiology, Yale University, New Haven, CT, USA and Center for Health Policy and Outcomes, Memorial Sloan Kettering Cancer Center, New York, NY, USA; 
${ }^{2}$ Institute of Health Policy, Management and Evaluation, Joint Centre for Bioethics, University of Toronto, Toronto, Ontario, Canada E-mail: fiona.miller@utoronto.ca

1 Bombard Y, Miller FA, Hayeems RZ et al: Health-care providers' views on pursuing reproductive benefit through newborn screening: the case of sickle cell disorders. Eur J Hum Genet 2012; 20: 498-504.

2 Ross LF: Newborn screening for sickle cell disease: whose reproductive benefit? Eur J Hum Genet 2012; 20: 484-485.

3 Health Council of the Netherlands: Screening: Between Hope and Hype. Hague: Health Council of the Netherlands, 2008.

4 Bailey Jr DB, Skinner D, Warren SF: Newborn screening for developmental disabilities: reframing presumptive benefit. Am J Public Health 2005; 95: 1889-1893.

5 Bailey DBJ, Beskow LM, Davis AM, Skinner D: Changing perspectives on the benefits of newborn screening. Ment Retard Dev Disabil 2006; 12: 10.

6 Alexander D, van Dyck PC: A vision of the future of newborn screening. Pediatrics 2006; 117: 350-354.

7 Bombard Y, Miller FA, Hayeems RZ, Avard D, Knoppers BM: Reconsidering reproductive benefit through newborn screening: a systematic review of guidelines on preconception, prenatal and newborn screening. Eur J Hum Genet 2010; 18: 751-760.

8 National Screening Committee: Second Report of the UK National Screening Committee. London: Department of Health, Social Services and Public Safety, 2000.

9 Botkin JR, Clayton EW, Fost NC et al: Newborn screening technology: proceed with caution. Pediatrics 2006; 117: 1793-1799.

10 Wilson JMG, Jungner G: Principles and Practice of Screening for Disease. Geneva: World Health Organization, 1968.

11 Bombard Y, Miller FA, Hayeems RZ et al: The expansion of newborn screening: is reproductive benefit an appropriate pursuit? Nat Rev Genet 2009; 10: 666-667.

12 Creighton S, Almqvist EW, MacGregor D et al: Predictive, pre-natal and diagnostic genetic testing for Huntington's disease: the experience in Canada from 1987 to 2000. Clin Genet 2003; 63: 462-475.

13 Ropka ME, Wenzel J, Phillips EK, Siadaty M, Philbrick JT: Uptake rates for breast cancer genetic testing: a systematic review. Cancer Epidemiol Biomarkers Prev 2006; 15: 840-855.

14 Sanderson SC, O'Neill SC, Bastian LA, Bepler G, McBride CM: What can interest tell us about uptake of genetic testing? Intention and behavior amongst smokers related to patients with lung cancer. Public Health Genomics 2010; 13: 116-124.

15 Mischler EH, Wilfond BS, Fost $\mathrm{N}$ et al: Cystic fibrosis newborn screening: impact on reproductive behavior and implications for genetic counseling. Pediatrics 1998; 102 : 44-52.

16 Ciske DJ, Haavisto A, Laxova A, Rock LZM, Farrell PM: Genetic counseling and neonatal screening for cystic fibrosis: an assessment of the communication process. Pediatrics 2001; 107: 699-705.

17 Wheeler PG, Smith R, Dorkin H, Parad RB, Comeau AM, Bianchi DW: Genetic counseling after implementation of statewide cystic fibrosis newborn screening: Two years' experience in one medical center. Genet Med 2001; 3: 411-415.

18 Ross LF, Clayton EW: Clinical and ethical considerations in managing carrier detection. Am J Public Health 2009; 99: 1348-1349.

19 Miller FA, Hayeems RZ, Robert JS: Carrier detection and clinical uncertainty: the case for public health ethics. Am J Public Health 2009; 99: 1349-1350.

20 Miller FA, Paynter M, Hayeems RZ et al: Understanding sickle cell carrier status identified through newborn screening: a qualitative study. Eur J Hum Genet 2010; 18: 303-308.

\section{$8 q 23.3$ and $11 q 23.1$ as modifying loci influencing the risk for CRC in Lynch syndrome}

European Journal of Human Genetics (2012) 20, 487-488; doi:10.1038/ejhg.2011.232; published online 14 December 2011

Recently, Houlle et al $^{1}$ reported results of two modifier SNPs in Lynch syndrome, rs16892766 on $8 \mathrm{q} 23.3$ and rs3802842 on $11 \mathrm{q} 23.1$ previously identified as low-susceptibility colorectal cancer (CRC) loci, challenging earlier reported findings. ${ }^{2,3}$

In 2009 Wijnen et al demonstrated that two SNPs located on $8 \mathrm{q} 23.3$ (rs16892766) and 11q23.1 (rs3802842) were associated with an increased risk of developing CRC in Dutch Lynch syndrome patients. The study revealed that patients' homozygote for SNP rs16892766 were associated with an elevated risk of CRC in a dose-dependent manner with a 2.16-fold increased risk of developing CRC, whereas the variant (CC) genotype of SNP rs3802842 was associated with an increased risk of $\mathrm{CRC}$ in female carriers only $(\mathrm{HR}=3.08){ }^{2}$ In a combined analysis of the two SNPs, the risk was significantly associated with the number of risk alleles and the effect was shown to be stronger in female carriers than in male carriers.

Recently, Talseth-Palmer et al ${ }^{3}$ confirmed the increased risk of CRC in Lynch syndrome patients in a combined Australian and Polish sample cohort but only in MLH1 mutation carriers. In this study the two Lynch syndrome populations (Australian and Polish) were analysed separately and together, as a larger sample size gives increased power and more reproducible results. SNP rs3802842 revealed a significant association on the risk of developing CRC in the combined sample population (Australian and Polish) with a HR of 2.67. When analysed separately, the Australian sample population displayed significant results whereas the Polish sample population displayed a trend, which demonstrates the increased power acquired when more samples are analysed together. SNP rs16892766 was only significantly associated with the increased risk of CRC in Australian MLH1 mutation carriers, but as this was the same SNP that displayed an association in the Dutch study ${ }^{2}$ we also analysed the additive effect of the two SNPs. We were able to show that MLH1 mutation carriers from Australia and Poland harbouring three risk alleles for the two SNPs developed CRC on average 24 years younger and were at 5.52-fold increased risk of CRC compared with individuals harbouring no risk alleles. The quote in the report by Houlle et al ' During the submission of this study, Talseth-Palmer et al ${ }^{15}$ reported that in $M L H 1$ carriers, but not in MSH2 carriers, the 11q23.1 CC and 8q23.3 AC genotypes were associated with an increased risk, but this significant association detected in 373 Australian mutation carriers was not found in 311 Polish mutation carriers analysed in the same study' is incorrect, as we did see this association in our combined sample cohort.

A decreased risk of CRC $(\mathrm{HR}=0.267, P=0.0271)$ for the $\mathrm{CC}$ (variant) genotype for SNP rs16892766 is reported by Houlle et al. ${ }^{1}$ According to previously published results discussed above this result is contradictory. A decreased risk of CRC indicate a later age of onset of CRC in the two individuals who harboured the CC genotype for SNP rs16892766, but the age of onset of CRC of these two individuals was not reported and can therefore not be commented on. It is highlighted by the French authors that the small number of subjects harbouring this genotype could affect the reported results. We believe that the observed results could be due to the fact that either of these two individuals harbours the variant (CC) genotype for SNP rs3802842, which seems to be important for the increased risk of CRC as observed in the Australian/Polish sample cohort.

A meta-analysis of the French and Dutch data set was performed by Houlle et $a l^{1}$ indicating that SNP rs3802842 at 11q23.1 is not associated with increased risk of developing CRC, and the only association observed in this meta-analysis was a decreased risk of CRC for SNP rs16892766 in male mutation carriers, which contradicts previously reported results. ${ }^{2,3}$ SNP rs16892766 (8q23.3) did not show an association with the CC (variant) genotype for the overall sample size as shown on the Forest plot (Figure $1^{1}$ ), which is not surprising as positive and negative results combined will end up with a neutral result. But false positive results in the Dutch study ${ }^{2}$ and false negative 